\title{
Enhanced Particle Swarm Optimization-Based Feeder Reconfiguration Considering Uncertain Large Photovoltaic Powers and Demands
}

\author{
Ying-Yi Hong, ${ }^{1}$ Faa-Jeng Lin, ${ }^{2}$ and Fu-Yuan Hsu ${ }^{1}$ \\ ${ }^{1}$ Department of Electrical Engineering, Chung Yuan Christian University, Chungli City 320, Taiwan \\ ${ }^{2}$ Department of Electrical Engineering, National Central University, Chungli City 320, Taiwan \\ Correspondence should be addressed to Ying-Yi Hong; yyh10632@yahoo.com.tw
}

Received 19 February 2014; Accepted 8 April 2014; Published 30 April 2014

Academic Editor: Ching-Song Jwo

Copyright ( $\odot 2014$ Ying-Yi Hong et al. This is an open access article distributed under the Creative Commons Attribution License, which permits unrestricted use, distribution, and reproduction in any medium, provided the original work is properly cited.

\begin{abstract}
The Kyoto protocol recommended that industrialized countries limit their green gas emissions in 2012 to $5.2 \%$ below 1990 levels. Photovoltaic (PV) arrays provide clear and sustainable renewable energy to electric power systems. Solar PV arrays can be installed in distribution systems of rural and urban areas, as opposed to wind-turbine generators, which cause noise in surrounding environments. However, a large PV array (several MW) may incur several operation problems, for example, low power quality and reverse power. This work presents a novel method to reconfigure the distribution feeders in order to prevent the injection of reverse power into a substation connected to the transmission level. Moreover, a two-stage algorithm is developed, in which the uncertain bus loads and PV powers are clustered by fuzzy-c-means to gain representative scenarios; optimal reconfiguration is then achieved by a novel mean-variance-based particle swarm optimization. The system loss is minimized while the operational constraints, including reverse power and voltage variation, are satisfied due to the optimal feeder reconfiguration. Simulation results obtained from a 70-bus distribution system with 4 large PV arrays validate the proposed method.
\end{abstract}

\section{Introduction}

The Kyoto protocol mandated that industrialized countries should reduce their greenhouse gas emissions in 2012 to $5.2 \%$ below their 1990 levels [1]. The Copenhagen Accord later called for the mitigation of global emissions in order to restrict rises in global temperatures to a maximum of $2^{\circ} \mathrm{C}[2]$. To attain a low carbon environment, utilizing renewable energy is essential to provide electric power in power systems. Of all renewable energies, wind-turbine generators (WTG), and solar photovoltaic $(\mathrm{PV})$ can provide the cleanest energy to consumers. Although a single unit size of WTG is generally much larger than that of a PV array, the former requires additional effort in terms of installation and maintenance. WTG is also limited by its produced noise, possibly damaging the ecosystem by altering the migration patterns of birds or other animals. However, solar PV arrays can provide noise-free energy, which may be installed in rural fields and rooftops of houses in urban areas. Solar PV has been widely studied in a power system, including solar power prediction [3], capacity sizing [4], and maximum power point tracking [5, 6]. These works focus only on solar PV modules without addressing system operation problems, which the penetration of solar PV energies in a distribution system causes (e.g., reverse power and high voltages at downstream customers).

Designed as interconnected networks, electric distribution networks are operated in a radial structure. The feeder configuration problem attempts to find a radial operating structure that optimizes network performance while satisfying operation constraints. In practice, distribution system operators may tend to reduce the system real-power loss and enhance voltage quality under normal conditions [7-12]. Jeon and Kim integrated tabu search with the simulated annealing algorithm to minimize system losses in [7]. The tabu search 
attempts to determine a solution using the greatest-descentlike algorithm which could not ensure any convergence property [7]. Lin et al. presented a refined genetic algorithm (RGA) to mitigate system losses [8]. Traditional crossover and mutation strategies were adjusted through a competition mechanism in RGA. Morton and Mareels presented a bruteforce solution to determine the status of each switch in order to minimize system loss in a radial distribution system [9]. The graph theory, which guarantees a global optimum yet requires an exhaustive search, was used in [9]. Wu and Tsai presented an approach based on particle swarm optimization (PSO) with an integer code to determine the switch operation schemes, which minimizes the system loss via the feeder reconfiguration in a distribution system [10]. Amanulla et al. presented a binary PSO-based algorithm to find the optimal statuses of the switches in order to maximize the reliability and minimize the real-power loss in a power system [11]. Hong and Ho proposed a Prufer-number-based genetic algorithm to reconfigure a network to ensure that the voltage drops caused by faults in voltage-sensitive areas are feasible [12]. Kavousi-Fard and Niknam proposed a selfadaptive modification method based on the clonal selection algorithm to reduce the cost of active power losses of the network and the customer interruption costs through the feeder reconfiguration [13]. Kavousi-Fard and Akbari-Zadeh used the shuffled frog leaping algorithm to reconfigure the network by considering three reliability indices and total system losses [14]. Kavousi-Fard et al. used a firefly algorithm to investigate the multiobjective probabilistic distribution feeder reconfiguration problem considering reliability [15].

The widely-installed renewable energy resources in the power system have issued new concerns regarding the feeder reconfiguration. Niknam et al. proposed a probabilistic method considering the uncertainty regarding the active and reactive load forecast errors as well as the wind-turbine output power variations concurrently to study the feeder reconfiguration. Self-adaptive modified teacher learning optimization algorithm was proposed to solve the multiobjective probabilistic problem [16]. Niknam et al. assessed the distribution feeder reconfiguration strategy, solved by a selfadaptive bat algorithm, and examined the effect of renewable energy sources on the reliability of the power system [17]. Niknam et al. proposed a two-stage approach: the roulette wheel mechanism in conjunction with the Weibull/Gaussian probability distribution functions of wind and demands were implemented to produce deterministic equivalents (scenarios); the feeder reconfiguration problem was then solved by the adaptive modified particle swarm optimization [18]. $\mathrm{Su}$ et al. presented a binary PSO-based method aiming at enhancing feeder configuration and reactive power control to accommodate more distributed generation resources [19]. Malekpour et al. presented an adaptive PSO algorithm to determine the optimal feeder reconfiguration while considering the uncertainty caused from the wind power generation and load demand [20]. The point estimate method was used to calculate the stochastic power flow [20]. Niknam et al. presented a honey bee mating optimization algorithm to investigate the distribution feeder reconfiguration problem while considering the effect of renewable energy sources on a system performance index [21].

Above literature survey reveals that system loss is a generally accepted performance index in the feeder reconfiguration problem. However, the above studies have the following limitations, which should be addressed in the modern smart grid:

(1) renewable generation resources. Although they were not considered in conventional feeder reconfiguration problems [3-15], they should be considered in modern smart distribution systems;

(2) uncertainty in renewable energies. Although uncertainty should be addressed in this area, owing to their intermittent characteristics, Kavousi-Fard and Niknam disregarded this essential factor [13, 21]. Although the uncertainty factor in renewable energies was considered in $[16,20]$, the point estimate method sampling two extra unsymmetrical operating points is considered to be less accurate than the Monte-Carlo simulation method $[16,20]$;

(3) coordination between VAR controllers and switches. To regulate the voltage profile in the distribution system, the statuses of all switches should incorporate with VAR controllers (inverters of distributed generation resources); however, most studies have ignored this factor [2-21];

(4) searching optimal statuses of switches: In contrast to genetic algorithms, tabu search, and other metaheuristic algorithms [3-9, 12-17, 21], PSO can search for the optimal solution efficiently. In addition to the encoding technique $[10,11,18-20]$, the search ap proach should also be improved to make the PSO algorithm more reliable and robust.

Therefore, this work presents a novel method based on the mean-variance concept of particles (i.e., possible solutions) in PSO to determine the optimal status of each switch in the distribution system. Uncertainties in the power generation from solar PV arrays and demands are clustered to become scenarios with different weighting factors. Restated, uncertainties in the problem are transformed into scenarios. The inverters of solar PV arrays are then fully utilized to coordinate with the switches to regulate the voltage profile in order to minimize the system losses using the interior-point algorithm. More specifically, rather than stochastic power flow studies, the regulated voltages of solar PV arrays in each scenario are adjusted by the interior-point-based optimal power flow with fixed switches determined by the enhanced PSO algorithm.

The rest of this paper is organized as follows. Section 2 introduces the assumptions in the studied problem and presents the mathematical formulation of the problem. Section 3 then describes the proposed method based on fuzzy-c-means and enhanced PSO algorithm. Section 4 summarizes the simulation results. Conclusions are finally drawn in Section 5, along with recommendations for future research. 


\section{Assumptions and Problem Formulation}

2.1. Assumptions. The following assumptions are made for the problem to avoid high voltages at the downstream customers and prevent the reverse power injected into the transmission level at the swing buses (substations).

(a) The status of each tie switch is determined one day ahead by the preventive control using forecasted PV powers and demands with uncertainties. The voltages of inverter-based solar PV arrays are evaluated as supplemental results to support the preventive control. These inverter-controlled voltages should be recomputed in the real-time corrective control. For the preventive control, the scenarios are predetermined based on the empirical experience. No control action for the tie switch is needed once the scenarios occur. That is, a predetermined network configuration is used for many scenarios. Thus, preventive control is highly applicable to uncertainty caused from the wind powers and demands.

(b) The inverter in either a rural field or an urban area at a bus can be considered as an aggregated one. Restated, hundreds of inverters of the solar PV arrays at a bus are operated with the same setting automatically or according to the commands of operators. Thus, a bus with large solar PV arrays is considered a voltagecontrolled bus rather than a constant PQ bus.

(c) The control center of a distribution system can communicate with the inverters through wire or wireless communication media in the demand management system (DMS) or SCADA. This assumption is applicable owing to the requirement of advanced ICT architecture in the smart distribution system.

(d) The DC voltage of a solar PV array is boosted to a higher DC voltage, which is generally supported by a capacitor. This higher DC voltage is then modulated to a nominal AC voltage via an inverter. To achieve the voltage regulation and prevent the injection of reverse power into the transmission level (swing buses), the DC bus between the boost converter and inverter must be supported by an energy storage; the inverter must be bidirectional as well. Alternatively, a capacitor is still used for the conventionally adopted inverter, and an additional inductor is used for an appended rectifier in parallel. When the PV arrays produce (absorb) the reactive power into (from) the power system, the corresponding bus operates with a lagging (leading) power factor.

2.2. Problem Formulation. As described in Section 1, an operator has many performance indices available to determine the switch statuses. Assume that the operator attempts to minimize the real-power losses and satisfy all operational constraints. In particular, no reverse power is injected into the transmission levels. Further, assume that more than one substation (between the transmission and distribution systems) acts as swing buses in the studied problem. According to the above four assumptions, the studied problem can be formulated as follows:

$$
\operatorname{Min} f(\widetilde{V}, \widetilde{\theta}, \widetilde{P}, \widetilde{Q}, x)
$$

s.t.

$$
\begin{gathered}
P M(\widetilde{V}, \widetilde{\theta}, \widetilde{P}, \widetilde{Q}, x)=0, \\
Q M(\widetilde{V}, \widetilde{\theta}, \widetilde{P}, \widetilde{Q}, x)=0, \\
V_{n}^{\min } \leq \widetilde{V}_{n} \leq V_{n}^{\max }, \quad n=1, \ldots, N, \\
I_{\ell}^{\min } \leq \widetilde{I}_{\ell} \leq I_{\ell}^{\max }, \quad \ell=1, \ldots, L, \\
\widetilde{P}_{s w}^{j} \geq 0, \quad j=1,2, \ldots, N S, \\
\left|\widetilde{P F}_{p v}\right| \geq P F^{\min }, \quad p v=1,2, \ldots, N P .
\end{gathered}
$$

The symbols are defined as follows:

$\underset{\text { tem }}{f(\widetilde{V}, \widetilde{\theta}, \widetilde{P}, \widetilde{Q}, x) \text { : stochastic real-power loss in the sys- }}$

$\operatorname{PM}(\widetilde{V}, \widetilde{\theta}, \widetilde{P}, \widetilde{Q}, x)$ : vector of the stochastic real-power balance equations

$\mathrm{Q} M(\widetilde{V}, \widetilde{\theta}, \widetilde{P}, \widetilde{Q}, x)$ : vector of the stochastic reactive power balance equations

$\widetilde{V}$ : vector of stochastic voltage magnitudes at all buses

$\widetilde{\theta}$ : vector of stochastic phase angles at all buses

$\widetilde{P}$ : vector of stochastic real-power injections at all buses

$\widetilde{Q}:$ vector of stochastic reactive power injections at all buses

$x$ : vector of statuses of all switches ( 1 or 0 )

$\widetilde{V}_{n}$ : stochastic voltage magnitude at bus $n, n=1, \ldots, N$

$\widetilde{I}_{\ell}:$ stochastic line flow at line $\ell, \ell=1, \ldots, L$

$\widetilde{P}_{s w}^{j}$ : total stochastic power injected into substation $j$, $j=1,2, \ldots, N S$

$\widetilde{P F}_{p v}:$ stochastic power factor at the $p v$ th photovoltaic array, $p v=1,2, \ldots, N P$

$N$ : number of system buses

$L:$ number of system lines

NS: number of substations (swing buses)

$N P$ : number of voltage controlled buses with solar PV arrays.

The values of components in $x$ must ensure that the structure of distribution system is radial, and no islands occur in the system. 
TABLE 1: Installed capacities of PV arrays and parameters of the Weibull distributions.

\begin{tabular}{lccc}
\hline Bus & $\begin{array}{c}\text { Installed } \\
\text { capacities }(\mathrm{kW})\end{array}$ & Scale parameter $(\kappa)$ & $\begin{array}{c}\text { Shape } \\
\text { parameter }(\gamma)\end{array}$ \\
\hline 13 & 750 & 0.35 & 2 \\
26 & 3000 & 0.35 & 2 \\
44 & 500 & 0.35 & 2 \\
61 & 4500 & 0.35 & 2 \\
\hline
\end{tabular}

\section{Proposed Method}

The mathematical formulation shown in (1)-(7) incorporated with the radial and nonislanding constraints is a stochastic mixed binary programming problem. This problem has difficulty in treating stochastic equality constraints in (2) and (3) and stochastic inequality constraints in (4)-(7). Thus, by using the fuzzy-c-means (FCM), the proposed method identifies the best representative scenarios with no random variables. This work presents a novel mean-variancebased PSO algorithm to determine the statuses of switches for all scenarios. Once the status of each switch is given, the nonstochastic representative scenarios can be solved using traditional robust nonlinear programming referred to herein as interior-point algorithm. By using the "fmincon" developed in MATLAB software, this work implements the interior-point algorithm. A detailed description of "fmincon" can be found in [22], which will be neglected herein.

3.1. Fuzzy-c-Means to Identify Representative Scenarios. Both $\mathrm{PV}$ power generations and demands are random variables with different stochastic distributions. Theoretically, the Monte-Carlo simulation can obtain an accurate solution in case the number of sampled PV power generations and demands from their stochastic distributions is sufficiently large. However, the Monte-Carlo simulation requires a very long CPU time. If these sampled PV power generations and demands can be clustered, then the center of each cluster can serve as a representative scenario. FCM attempts to identify the representative scenarios obtained from all random variables with their individual stochastic distributions. Restated, the scenarios with real values are generated according to the stochastic distributions of all random variables (PV power generations and demands). The data sets in these scenarios are clustered using FCM. Namely, the center vector in each cluster refers to the representative scenario, and the sum of membership values in each cluster indicates the corresponding weighting factor for this representative scenario.

Bezdek et al. defined $J(U, V)$ as an objective function in the FCM algorithm [23] as follows:

$$
J(U, V)=\sum_{i=1}^{M} \sum_{c=1}^{C}\left(\mu_{c i}\right)^{m}\left\|X_{i}-V_{c}\right\|^{2}, \quad 1 \leq m<\infty,
$$

where $C$ signifies the clustering number, which is determined in Section 3.2, and is fixed for in FCM. Additionally, $M$ denotes the number of data sets generated from the MonteCarlo simulation herein; $V_{c}$ refers to the vector of the center in the $c$ th cluster; $X_{i}$ denotes the $i$ th data vector for clustering; the membership function value $\mu_{c i}$ serves as a weighting factor between $V_{c}$ and $X_{i}$. After the minimum of $J(U, V)$ is gained, the $M$ data vectors are partitioned into $C$ clusters. Let symbols $h$ and $\varepsilon$ represent the iterative index and convergence tolerance, respectively. Bezdek developed the following four algorithmic steps to attain the optimum.

Step 1. Initialize a matrix of membership functions as follows:

$$
U^{(h)}=\left[\mu_{c i}^{(h)}\right] \in R^{C \times M},
$$

where initial $h$ equals 0 .

Step 2. Let $h=h+1$. Compute the center of the $c$ th cluster as follows:

$$
V_{c}=\frac{\sum_{i=1}^{M}\left(\mu_{c i}^{(h)}\right)^{m} X_{i}}{\sum_{i=1}^{M}\left(\mu_{c i}^{(h)}\right)^{m}} \quad 1 \leq c \leq C, 1 \leq i \leq M .
$$

Step 3. Update $\mu_{c i}^{(h)}$ for all $X_{i}, i=1, \ldots, M$.

Consider

$$
\mu_{c i}^{(h)}=\frac{1}{\sum_{j=1}^{C}\left[\left\|X_{i}-V_{c}\right\| /\left\|X_{i}-V_{j}\right\|\right]^{2 /(m-1)}} .
$$

Step 4. If $\left\|U^{(h)}-U^{(h-1)}\right\|<\varepsilon$, stop; otherwise, go to Step 2.

3.2. Optimal Number of Clusters. Yang and $\mathrm{Wu}$ proposed a partition separation (PS) validity index, in which a normalized partition coefficient is aggregated with an exponential separation measure for each cluster [24].

The PS validity index for cluster $c$ is defined as follows:

$$
\operatorname{PS}(c)=\sum_{i=1}^{M} \frac{\mu_{c i}^{2}}{\mu^{\max }}-\exp \left(-\frac{\min _{c \neq k}\left\{\left\|V_{c}-V_{k}\right\|^{2}\right\}}{\beta_{T}}\right),
$$

where $\mu^{\max }=\max _{1 \leq c \leq C}\left\{\sum_{i=1}^{M} \mu_{c i}^{2}\right\}, \beta_{T}=\left(\sum_{c=1}^{C}\left\|V_{c}-\bar{V}\right\|^{2}\right) / C$, and $\bar{V}$ denotes the mean of all $V_{c}$ s.

The PS validity index for $C$ clusters is defined as follows:

$$
\operatorname{PS}(C)=\sum_{c=1}^{C} \operatorname{PS}(c)
$$

A large PS $(C)$ refers to a situation in which cluster $c$ is compact inside and far away from other $(C-1)$ clusters. Consequently, the optimal number $C^{*}$ (i.e., $C$ defined in Section 3.1) of clusters can be obtained as follows:

$$
C^{*}=\max _{2 \leq C \leq \sqrt{M}} \operatorname{PS}(C) .
$$

3.3. Mean-Variance-Based PSO Algorithm to Determine $x$. As an evolutionary optimization method, PSO minimizes an objective function (performance index) by imitating 
TABLE 2: PS validity indices for different numbers of clusters.

\begin{tabular}{lccccccccc}
\hline C & 2 & 3 & 4 & 5 & 6 & 7 & 8 & 9 & 10 \\
\hline PS & 1.687 & 2.245 & 2.434 & 2.283 & 2.232 & 1.875 & 3.039 & 0.886 & 2.388 \\
\hline
\end{tabular}
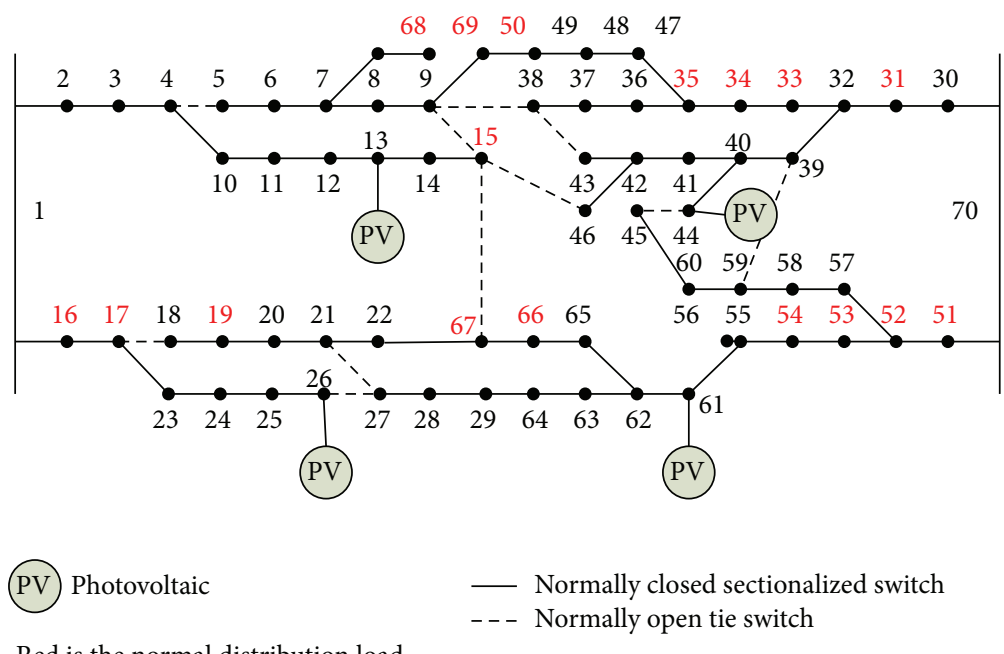

Red is the normal distribution load

Black is the fixed load

FIgURE 1: One-line diagram for the 70-bus distribution system.

the behavior of schooling fish or flocking birds [25, 26]. PSO comprises a population of particles (individuals) that represent possible solutions moving in a $\psi$-dimensional search space.The iterative temporary solution and updated value of a particle $p$ are called the position and velocity, respectively. Let vectors $x_{p}$ and $\Delta x_{p}$ be the $\psi$-dimensional position and velocity of particle $p, p=1,2, \ldots, P$ (i.e., number of population size), respectively.

The proposed method is based on the mean of the particles and standard deviation (squared root of variance) of the distances between any two particles in the $t$ th iterations. The updated value of a particle $p$ at iteration $t$ is expressed as follows:

$$
\begin{aligned}
\Delta x_{p}^{t+1}= & w^{t} \Delta x_{p}^{t}+a^{t} r_{1}^{t}\left(p_{\text {best }}^{t}-x_{p}^{t}\right) \\
& +b^{t} r_{2}^{t}\left(g_{\text {best }}^{t}-x_{p}^{t}\right)+c^{t} r_{3}^{t}\left(g_{\text {best }}^{t}-\bar{x}_{p}^{t}\right),
\end{aligned}
$$

where the inertia weight $w^{t}$ is decreased linearly from 0.5 to 0.3 because a large $w^{t}$ is used for global searches, whereas local searches require a small $w^{t}$. The symbols $r_{1}, r_{2}$, and $r_{3}$ are random numbers within $[0,1]$. Additionally, $p_{\text {best }}^{t}$ and $g_{\text {best }}^{t}$ are the best position of a particle in the iteration $t$ and the best known position up to present, respectively. The learning factors $a$ and $b$ should meet $a+b \leq 4[25,26]$ and are defined as

$$
a^{t}=b^{t}=1+\frac{1}{\left(1+\exp \left(f\left(g_{\text {best }}^{1}\right) / f\left(g_{\text {best }}^{t}\right)\right)\right)} \text {. }
$$

Since the last term on the right hand side of (15) is appended, compared to the original PSO, $c^{t}=4-a^{t}-b^{t}$. Also, $\bar{x}_{p}^{t}$ is a $\psi$-dimensional vector of the mean values of all particles. The last term introduced in (15) is used to coordinate the global and local searches.

Let $\sigma^{t}$ be the standard deviation of all distances among particles in the $t$ th iteration. If all distances follow a Gaussian distribution, then $\bar{x}_{p}^{t} \pm 3 \sigma^{t}$ covers $99.7 \%$ of all particles. Let $\eta^{t}=3-2 c^{t}$. Because $c^{t}$ in (15) decreases to zero, the values of $\eta^{t}$ are increased to 3 . This work recommends that the particles outside the distance of $\bar{x}_{p}^{t} \pm \eta^{t} \sigma^{t}$ in iteration $t$ should be discarded. These discarded particles are substituted by $g_{\text {best }}^{t}$. Restated, if $x_{p}^{t}$ is inside the $\bar{x}_{p}^{t} \pm \eta^{t} \sigma^{t}$, then

$$
x_{p}^{t+1}=x_{p}^{t}+\Delta x_{p}^{t}
$$

If $x_{p}^{t}$ is outside $\bar{x}_{p}^{t} \pm \eta^{t} \sigma^{t}$, then

$$
x_{p}^{t+1}=g_{\text {best }}^{t}+\Delta x_{p}^{t}
$$

In this work, $x_{p}^{t+1}$ denotes the vector of statuses of all switches. All elements of $x_{p}^{t+1}$ are bounded to 0 or 1 through the sigmoid function. Because the number of switches with "closed" statuses is fixed (say $\xi$ ) for a distribution system to ensure a radial structure, the largest $\xi$ elements in $x$ are rounded to 1 and the remaining elements are reset to 0 . 


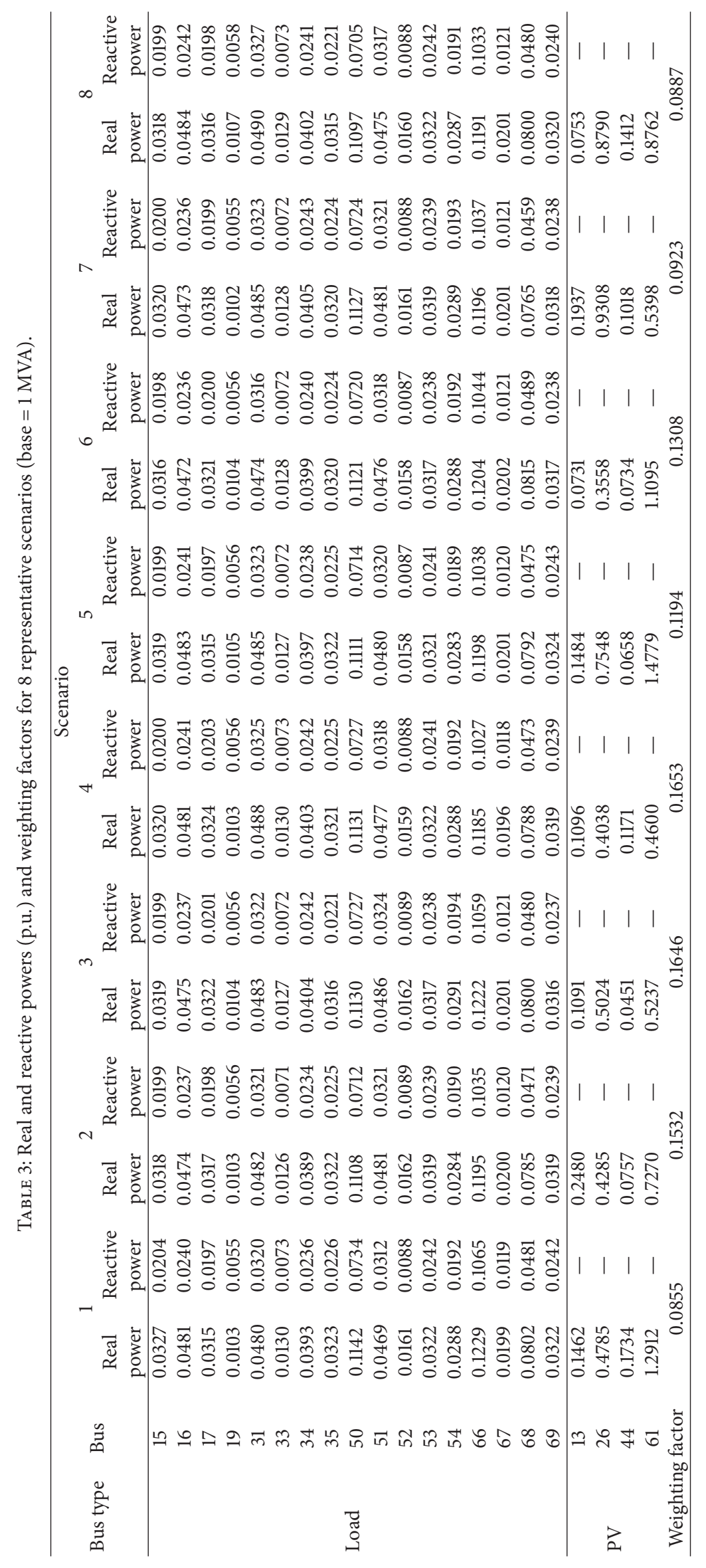


TABLE 4: Optimal results before feeder reconfiguration.

\begin{tabular}{|c|c|c|c|c|c|c|c|c|}
\hline Scenario & 1 & 2 & 3 & 4 & 5 & 6 & 7 & 8 \\
\hline \multicolumn{9}{|c|}{ Voltage magnitude (p.u.) } \\
\hline $\mathrm{V}_{1}$ & 1.041 & 1.043 & 1.039 & 1.045 & 1.026 & 1.047 & 0.974 & 1.019 \\
\hline $\mathrm{V}_{13}$ & 1.021 & 1.034 & 1.015 & 1.021 & 1.006 & 1.020 & 0.950 & 0.991 \\
\hline $\mathrm{V}_{26}$ & 1.050 & 1.050 & 1.050 & 1.050 & 1.050 & 1.050 & 0.984 & 1.050 \\
\hline $\mathrm{V}_{44}$ & 1.015 & 1.010 & 1.008 & 1.012 & 1.006 & 1.009 & 1.024 & 1.014 \\
\hline $\mathrm{V}_{61}$ & 1.049 & 1.000 & 0.980 & 0.974 & 1.050 & 1.035 & 1.009 & 1.014 \\
\hline $\mathrm{V}_{70}$ & 1.050 & 1.050 & 1.050 & 1.050 & 1.047 & 1.050 & 1.050 & 1.050 \\
\hline \multicolumn{9}{|c|}{ Reactive power (kVAr) } \\
\hline $\mathrm{Q}_{13}$ & 109.684 & 185.963 & 81.792 & 82.203 & 111.297 & 54.847 & 0.002 & 56.510 \\
\hline $\mathrm{Q}_{26}$ & 207.808 & 209.497 & 206.809 & 206.289 & 209.705 & 204.487 & -361.796 & 207.732 \\
\hline $\mathrm{Q}_{44}$ & 130.072 & 56.783 & 33.835 & 87.807 & 49.377 & 55.037 & 502.178 & 105.918 \\
\hline $\mathrm{Q}_{61}$ & 941.614 & 545.250 & 392.784 & 345.034 & 819.679 & 832.136 & 954.342 & 657.163 \\
\hline \multicolumn{9}{|c|}{ Real power at swing bus (kW) } \\
\hline $\mathrm{P}_{1}$ & 361.171 & 304.607 & 375.732 & 473.122 & 91.277 & 557.162 & -95.092 & 50.501 \\
\hline $\mathrm{P}_{70}$ & 1953.526 & 2640.701 & 2912.282 & 2902.069 & 1877.819 & 2241.501 & 2800.633 & 2410.641 \\
\hline Total load $(\mathrm{kW})$ & 4286.679 & 4276.304 & 4285.446 & 4281.481 & 4280.026 & 4281.264 & 4278.722 & 4279.626 \\
\hline Loss $(\mathrm{kW})$ & 117.429 & 148.208 & 182.827 & 184.226 & 135.961 & 129.235 & 193.034 & 153.292 \\
\hline \multicolumn{9}{|c|}{ Aggregating loss $=157.874 \mathrm{~kW}$} \\
\hline
\end{tabular}

TABLE 5: Optimal results after the feeder reconfiguration using the proposed method.

\begin{tabular}{|c|c|c|c|c|c|c|c|c|}
\hline Scenario & 1 & 2 & 3 & 4 & 5 & 6 & 7 & 8 \\
\hline \multicolumn{9}{|c|}{ Voltage magnitude (p.u.) } \\
\hline $\mathrm{V}_{1}$ & 1.050 & 1.050 & 1.050 & 1.050 & 1.046 & 1.050 & 1.037 & 1.040 \\
\hline $\mathrm{V}_{13}$ & 1.013 & 1.024 & 1.008 & 1.009 & 1.009 & 1.004 & 1.004 & 0.994 \\
\hline $\mathrm{V}_{26}$ & 1.036 & 1.032 & 1.038 & 1.030 & 1.050 & 1.026 & 1.050 & 1.050 \\
\hline $\mathrm{V}_{44}$ & 0.994 & 1.014 & 1.012 & 1.016 & 0.978 & 0.998 & 1.015 & 1.014 \\
\hline $\mathrm{V}_{61}$ & 1.050 & 1.047 & 1.029 & 1.023 & 1.050 & 1.050 & 1.030 & 1.050 \\
\hline $\mathrm{V}_{70}$ & 1.025 & 1.050 & 1.050 & 1.050 & 1.016 & 1.035 & 1.050 & 1.046 \\
\hline \multicolumn{9}{|c|}{ Reactive power (kVAr) } \\
\hline $\mathrm{Q}_{13}$ & 109.686 & 185.963 & 81.792 & 82.204 & 111.298 & 54.847 & 145.300 & 56.510 \\
\hline $\mathrm{Q}_{26}$ & 358.888 & 321.407 & 376.783 & 302.842 & 415.400 & 266.856 & 419.470 & 411.446 \\
\hline $\mathrm{Q}_{44}$ & 130.073 & 56.783 & 33.835 & 87.807 & 49.378 & 55.037 & 76.380 & 105.918 \\
\hline $\mathrm{Q}_{61}$ & 541.639 & 545.250 & 392.784 & 345.034 & 532.708 & 530.894 & 404.858 & 532.886 \\
\hline \multicolumn{9}{|c|}{ Real power at swing bus (kW) } \\
\hline $\mathrm{P}_{1}$ & 1209.57 & 1149.71 & 1223.55 & 1324.58 & 928.005 & 1415.21 & 702.954 & 885.934 \\
\hline $\mathrm{P}_{70}$ & 1081.67 & 1726.06 & 1974.25 & 1959.51 & 1016.86 & 1356.16 & 1893.88 & 1508.39 \\
\hline Loss $(\mathrm{kW})$ & 93.979 & 78.674 & 92.627 & 93.126 & 111.735 & 101.945 & 84.322 & 86.471 \\
\hline \multicolumn{9}{|c|}{ Aggregating loss $=92.875 \mathrm{~kW}$} \\
\hline
\end{tabular}

After the status of each switch is determined using the mean-variance-based PSO, the topology of a distribution system is fixed. Additionally, the uncertainties in wind powers and demands are modeled by many scenarios with real numbers obtained by FCM. Thus, each scenario becomes a traditional nonlinear "optimal power flow" (OPF) problem, which adjusts the inverter-controlled voltages to minimize the weighting real-power losses and meets the operational constraints, including the reverse power. The weighting factor of the objective function for each scenario $c$ is evaluated by $\sum_{i} \mu_{c i}$ where the $i$ th data set belongs to the $c$ th cluster. In this work, the optimal power flow problem is solved using the interior-point algorithm developed in MATLAB software.

\section{Simulation Results}

A case study involving a 70-bus distribution system is used as an example [27], as shown in Figure 1, to show the simulation 
TABLE 6: Optimal results after feeder reconfiguration using chaos-based PSO and GA.

\begin{tabular}{|c|c|c|c|c|c|c|c|c|}
\hline Scenario & 1 & 2 & 3 & 4 & 5 & 6 & 7 & 8 \\
\hline \multicolumn{9}{|c|}{ Voltage magnitude (p.u.) } \\
\hline $\mathrm{V}_{1}$ & 1.050 & 1.050 & 1.050 & 1.050 & 1.046 & 1.050 & 1.037 & 1.040 \\
\hline $\mathrm{V}_{13}$ & 1.019 & 1.030 & 1.015 & 1.015 & 1.015 & 1.011 & 1.011 & 1.000 \\
\hline $\mathrm{V}_{26}$ & 1.036 & 1.032 & 1.038 & 1.030 & 1.050 & 1.026 & 1.050 & 1.050 \\
\hline $\mathrm{V}_{44}$ & 0.992 & 1.011 & 1.009 & 1.014 & 0.976 & 0.995 & 1.013 & 1.011 \\
\hline $\mathrm{V}_{61}$ & 1.050 & 1.047 & 1.029 & 1.023 & 1.050 & 1.050 & 1.030 & 1.050 \\
\hline $\mathrm{V}_{70}$ & 1.026 & 1.050 & 1.050 & 1.050 & 1.017 & 1.035 & 1.050 & 1.046 \\
\hline \multicolumn{9}{|c|}{ Reactive power (kVAr) } \\
\hline $\mathrm{Q}_{13}$ & 109.686 & 185.963 & 81.792 & 82.204 & 111.298 & 54.847 & 145.300 & 56.510 \\
\hline $\mathrm{Q}_{26}$ & 358.889 & 321.407 & 376.783 & 302.842 & 418.678 & 266.856 & 422.320 & 415.772 \\
\hline $\mathrm{Q}_{44}$ & 130.073 & 56.783 & 33.835 & 87.807 & 49.378 & 55.037 & 76.380 & 105.918 \\
\hline $\mathrm{Q}_{61}$ & 536.509 & 545.250 & 392.784 & 345.034 & 526.576 & 525.289 & 404.859 & 528.011 \\
\hline \multicolumn{9}{|c|}{ Real power at swing bus $(\mathrm{kW})$} \\
\hline $\mathrm{P}_{1}$ & 1145.56 & 1087.05 & 1159.05 & 1260.09 & 864.00 & 1350.20 & 639.51 & 820.838 \\
\hline $\mathrm{P}_{70}$ & 1146.37 & 1791.07 & 2039.45 & 2024.28 & 1082.32 & 1421.35 & 1958.74 & 1573.05 \\
\hline Loss $(\mathrm{kW})$ & 94.658 & 81.017 & 93.310 & 93.400 & 113.189 & 102.117 & 85.737 & 86.035 \\
\hline \multicolumn{9}{|c|}{ Aggregating loss $=93.738 \mathrm{~kW}$} \\
\hline
\end{tabular}

results obtained by the proposed method. Buses 1 and 70 are two substations (the swing buses). The solid lines denote the normally closed sectionalized switches, and the dot lines are normally open tie switches. Totally, the statuses of 79 switches must be determined in this work by the proposed meanvariance-based PSO.

Four large solar PV arrays are installed at buses 13, 26, 44, and 61. Characteristics of the solar PV arrays are the Weibull distributions; that is, pdf $(\mathrm{Pg})=(\kappa / \gamma)(\mathrm{Pg} / \gamma) \kappa-1$ $\exp (-(\mathrm{Pg} / \gamma) \kappa)$, where $\mathrm{Pg}$ denotes the PV generation. Table 1 lists the installed capacities of all PV arrays and their parameters of the Weibull distributions.

In this system, the loads at 17 buses are modeled by the Gaussian distributions. They are buses 15-17, 19, 31, 33-35, $50-54$, and 66-69. The mean and standard deviation of each stochastic load are 0.8 p.u. and $5 \%$ (based on its original MVA), respectively. The loads at other buses are fixed.

Three hundred sets of PV generations and loads are randomly produced using the parameters of the Weibull and Gaussian distributions, respectively; that is, $M=300$, in this work. Next, by using FCM, these 300 sets of data are clustered together by considering different $C$. Theoretically, the maximum number of clusters is $\sqrt{300}(\cong 17$, see (14)). However, the computational burden in a PC is considered here; let the maximum number of clusters be 10 . The total CPU time $(C=2,3, \ldots, 10)$ to cluster these sampled PV generations and loads is $1.38 \mathrm{~s}$, which was estimated by a PC with Intel Core i5 $2.50 \mathrm{GHz}$ and $8 \mathrm{~GB}$ RAM. Table 2 lists the PS validity indices for $C=2,3, \ldots, 10$. This table reveals that the optimal clustering number is 8 (i.e., $C^{*}=8$ in (14)) because its corresponding PS validity value (3.039) is the largest.

The 8 representative scenarios obtained by FCM were examined first by running OPF. The voltage profile and reactive powers were adjusted using 4 inverter-based solar PV at buses 13, 26, 44, and 61. Table 3 lists the 17 load buses with their representative real- and reactive-power loads (p.u.) in the 8 representative scenarios. The 4 solar PV arrays generate real power only initially with the unity power factor. This table also shows the weighting factor obtained by FCM for each scenario.

First, the interior-point-based OPF was used to regulate the voltage profile and minimize the system losses individually for these 8 scenarios. Table 4 summarizes the optimal results before the feeder reconfiguration. This table reveals that a reverse power $-95.092 \mathrm{~kW}$ was injected into bus 1 in scenario 7 . There is also reverse reactive power $(-361.796 \mathrm{kVAr})$ at bus 26 . The other scenarios had no reverse power at buses 1 and 70 .

By using the proposed method, the statuses of 79 switches were determined using the mean-variance-based PSO; the voltage profile was also regulated using the interior-pointbased OPF. The population size was 10 , and the inertia was decreased linearly from 0.5 to 0.3 in 100 iterations in the proposed PSO algorithm. Table 5 lists the optimal solutions obtained by the proposed method. The injection powers -95.092 (bus 1) and 2,800.633 kW (bus 70) before feeder reconfiguration were changed to 702.954 (bus 1) and $1,893.876 \mathrm{~kW}$ (bus 70) after feeder reconfiguration in scenario 7 , respectively. The system losses were also reduced from 157.874 to $92.875 \mathrm{~kW}$. The proposed method required $11,835.41$ $\mathrm{CPU}$ seconds to attain the optimal solution. Figure 2 shows the optimal feeder reconfiguration using the proposed method.

Table 6 shows the optimal solutions gained by using chaos-based PSO and GA. The results gained by chaos-based PSO and GA are the same. The numerical parameters used in the chaos-based PSO are the same as those in the proposed method. The crossover and mutation rates in GA are 


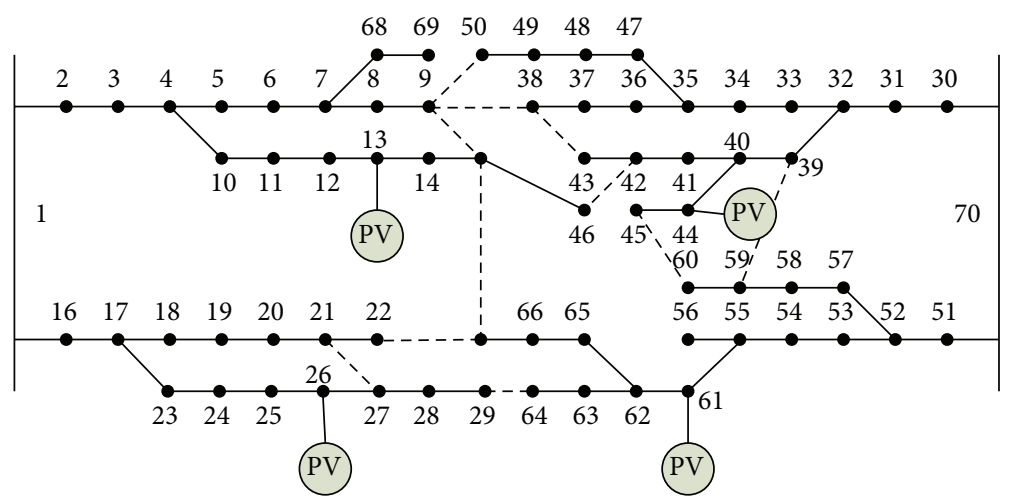

Figure 2: Optimal feeder reconfiguration using the proposed method.

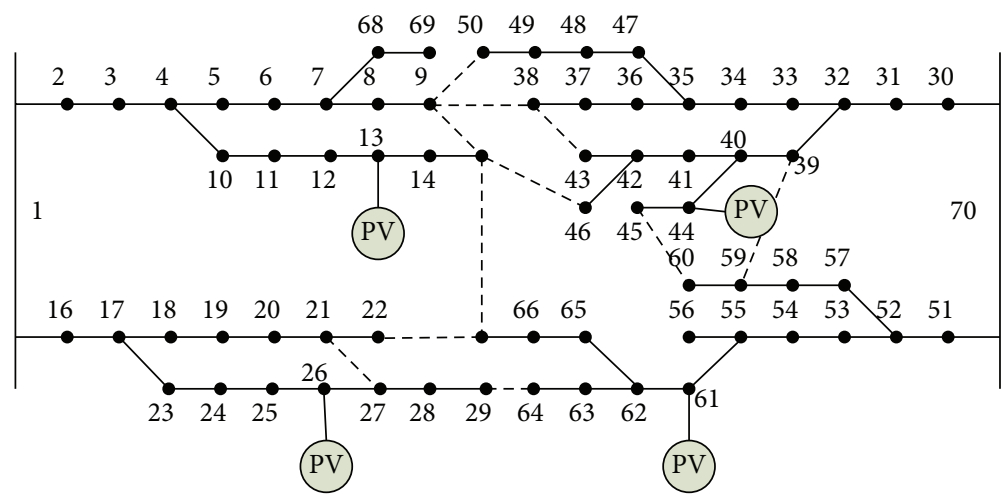

FIGURE 3: Optimal feeder reconfiguration obtained by chaos-based PSO and GA.

0.8 and $1 / 790$, respectively. The merits of the proposed method are as follows. (a) The CPU times required by chaosbased PSO $(15,224.55 \mathrm{~s})$ and GA $(18,508.64 \mathrm{~s})$ are much longer than that $(11,835.41 \mathrm{~s})$ required in the proposed method. (b) The aggregating loss attained by the proposed method is $92.875 \mathrm{~kW}$, which is smaller than $93.738 \mathrm{~kW}$ obtained by both chaos-based PSO and GA. Figure 3 illustrates the optimal feeder reconfiguration obtained by the chaos-based PSO and GA algorithms.

\section{Conclusions}

This work presents a novel method that incorporates FCM, partition separation validity index, mean-variance-based PSO, and interior-point-based optimal power flow to conduct feeder reconfiguration in order to prevent the revere power caused by uncertain solar PV arrays from injecting into the transmission level. The novelties are as follows: (a) the uncertainty in the PV powers and demands is modeled by multiple scenarios gained by FCM in order to avoid probabilistic calculations; (b) the novel mean-variance-based PSO implements an adaptive elite strategy that replaces the particles far away from $g_{\text {best }}^{t}$ with $g_{\text {best }}^{t}$. The number of replacement decreases according to the standard deviation of distances among particles. Simulation results demonstrate that the proposed method is faster than the chaos-based PSO method. Furthermore, the proposed method can solve the problem with the reverse power and high voltage at the customers' side while minimizing the system loss.

\section{Conflict of Interests}

The authors declare that there is no conflict of interests regarding the publication of this paper.

\section{Acknowledgments}

The authors would like thank the National Science Council of the Republic of China, Taiwan, for financially supporting this research under Contract no. NSC103-3113-P-008-001. Ted Knoy is appreciated for his editorial assistance.

\section{References}

[1] "Kyoto Protocol to the United Nations-framework convention on climate change," December 1997.

[2] The Copenhagen Accord, "United Nations Climate Change Conference," December 2009.

[3] V. L. Brano, G. Ciulla, and M. D. Falco, "Artificial neural networks to predict the power output of a PV panel," International Journal of Photoenergy, vol. 2014, Article ID 193083, 12 pages, 2014. 
[4] M. Engin, "Sizing and simulation of PV-wind hybrid power system," International Journal of Photoenergy, vol. 2013, Article ID 217526, 10 pages, 2013.

[5] K. H. Chao, L. Y. Chang, and H. C. Liu, "Maximum power point tracking method based on modified particle swarm optimization for photovoltaic systems," International Journal of Photoenergy, vol. 2013, Article ID 583163, 6 pages, 2013.

[6] H. Mahamudul, M. Saad, and M. I. Henk, "Photovoltaic system modeling with fuzzy logic based maximum power point tracking algorithm," International Journal of Photoenergy, vol. 2013, Article ID 762946, 10 pages, 2013.

[7] Y. J. Jeon and J. C. Kim, "Network reconfiguration in radial distribution system using simulated annealing and tabu search," in IEEE PES Winter Meeting, pp. 23-27, January 2000.

[8] W.-M. Lin, F.-S. Cheng, and M.-T. Tsay, "Distribution feeder reconfiguration with refined genetic algorithm," IEE Proceedings: Generation, Transmission and Distribution, vol. 147, no. 6, pp. 349-354, 2000.

[9] A. B. Morton and I. M. Y. Mareels, "An efficient brute-force solution to the network reconfiguration problem," IEEE Transactions on Power Delivery, vol. 15, no. 3, pp. 996-1000, 2000.

[10] W.-C. Wu and M.-S. Tsai, "Application of enhanced integer coded particle swarm optimization for distribution system feeder reconfiguration," IEEE Transactions on Power Systems, vol. 26, no. 3, pp. 1591-1599, 2011.

[11] B. Amanulla, S. Chakrabarti, and S. N. Singh, "Reconfiguration of power distribution systems considering reliability and power loss," IEEE Transactions on Power Delivery, vol. 27, no. 2, pp. 918-926, 2012.

[12] Y.-Y. Hong and S.-Y. Ho, "Determination of network configuration considering multiobjective in distribution systems using genetic algorithms," IEEE Transactions on Power Systems, vol. 20, no. 2, pp. 1062-1069, 2005.

[13] A. Kavousi-Fard and T. Niknam, "Optimal distribution feeder reconfiguration for reliability improvement consideringuncertainty," to appear in IEEE Transactions on Power Delivery.

[14] A. Kavousi-Fard and M. R. Akbari-Zadeh, "Reliability enhancement using optimal distribution feeder reconfiguration," Neurocomputing, vol. 106, pp. 1-11, 2013.

[15] A. Kavousi-Fard, T. Niknam, and A. Bazia, "A novel multi-objective self-adaptive modified firefly algorithm for optimal operation management of stochastic DFR strategy," International Transactions on Electrical Energy Systems, 2014.

[16] T. Niknam, A. Kavousi Fard, and A. Baziar, "Multi-objective stochastic distribution feeder reconfiguration problem considering hydrogen and thermal energy production by fuel cell power plants," Energy, vol. 55, pp. 680-691, 2014.

[17] T. Niknam, A. Kavousi Fard, and A. Baziar, "Multi-objective stochastic distribution feeder reconfiguration problem considering hydrogen and thermal energy production by fuel cell power plants," Energy, vol. 64, pp. 342-354, 2014.

[18] T. Niknam, A. Kavousifard, and J. Aghaei, "Scenario-based multiobjective distribution feeder reconfiguration considering wind power using adaptive modified PSO," IET Renewable, vol. 6, no. 4, pp. 236-247, 2012.

[19] S.-Y. Su, C.-N. Lu, R.-F. Chang, and G. Gutiérrez-Alcaraz, "Distributed generation interconnection planning: a wind power case study," IEEE Transactions on Smart Grid, vol. 2, no. 1, pp. 169-177, 2011.
[20] A. R. Malekpour, T. Niknam, A. Pahwa, and A. Kavousi-Fard, "Multi-objective stochastic distribution feeder reconfiguration in systems with wind power generators and fuel cells using the point estimate method," IEEE Transactions on Power Systems, vol. 28, no. 2, pp. 1483-1492, 2013.

[21] T. Niknam, A. K. Fard, and A. Seifi, "Distribution feeder reconfiguration considering fuel cell/wind/photovoltaic power plants," Renewable Energy, vol. 37, no. 1, pp. 213-225, 2012.

[22] Optimization Toolbox-Fmincon, MATLAB, the MathWorks, 2009.

[23] J. C. Bezdek, R. Ehrlich, and W. Full, "FCM: the fuzzy c-means clustering algorithm," Computers and Geosciences, vol. 10, no. 23, pp. 191-203, 1984.

[24] M.-S. Yang and K.-L. Wu, "A new validity index for fuzzy clustering," in Proceedings of the 10th IEEE International Conference on Fuzzy Systems, pp. 89-92, Melbourne, Australia, December 2001.

[25] J. Kennedy and R. Eberhart, Swarm Intelligence, Morgan Kaufmann Publishers, Elsevier, San Francisco, Calif, USA, 2001.

[26] R. Eberhart and J. Kennedy, "New optimizer using particle swarm theory," in Proceedings of the 6th International Symposium on Micro Machine and Human Science, pp. 39-43, October 1995.

[27] A. R. Malekpour and T. Niknam, "A probabilistic multi-objective daily Volt/Var control at distribution networks including renewable energy sources," Energy, vol. 36, no. 5, pp. 3477-3488, 2011. 

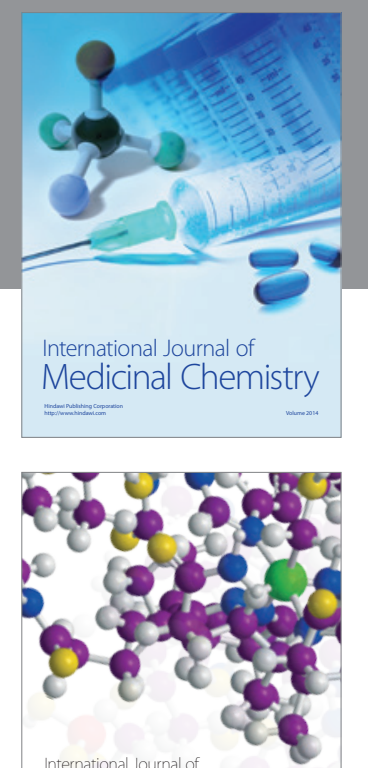

\section{Carbohydrate} Chemistry

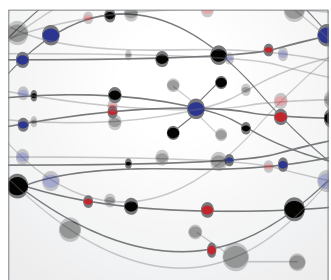

The Scientific World Journal
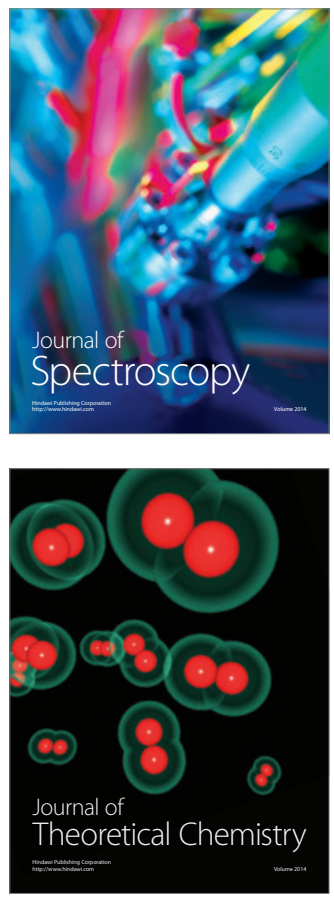
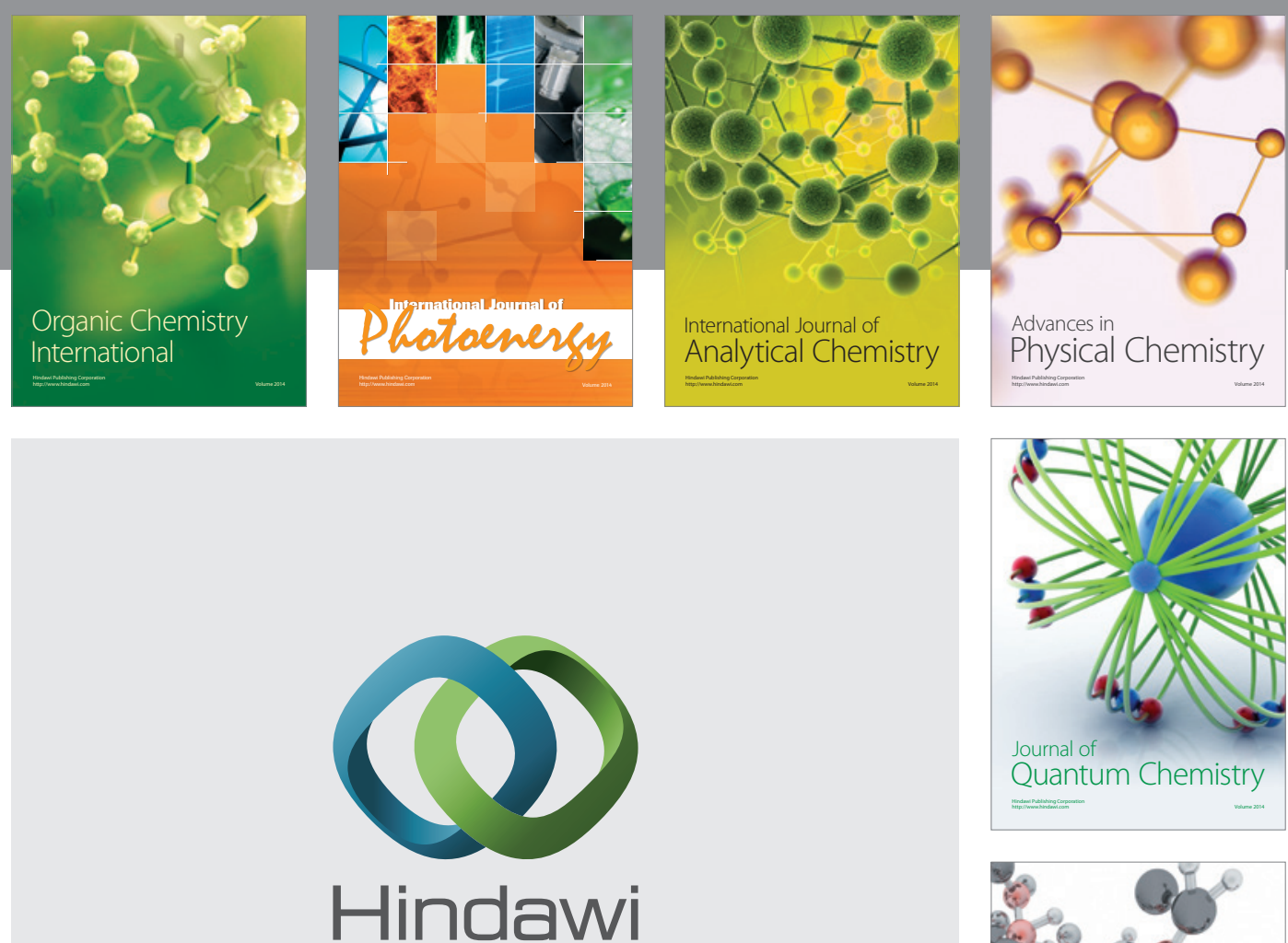

Submit your manuscripts at

http://www.hindawi.com

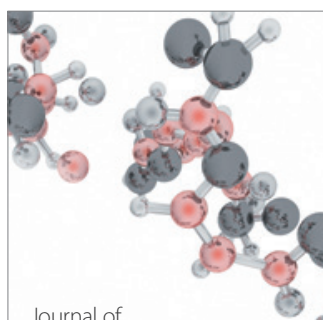

Analytical Methods

in Chemistry

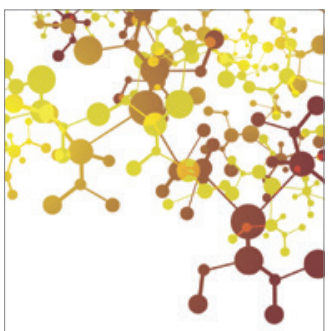

Journal of

Applied Chemistry

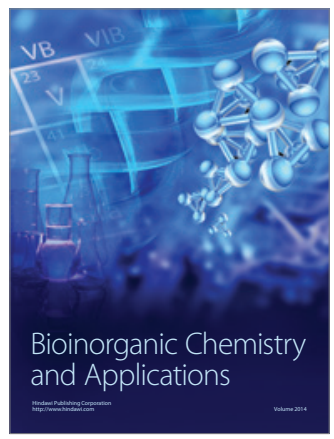

Inorganic Chemistry
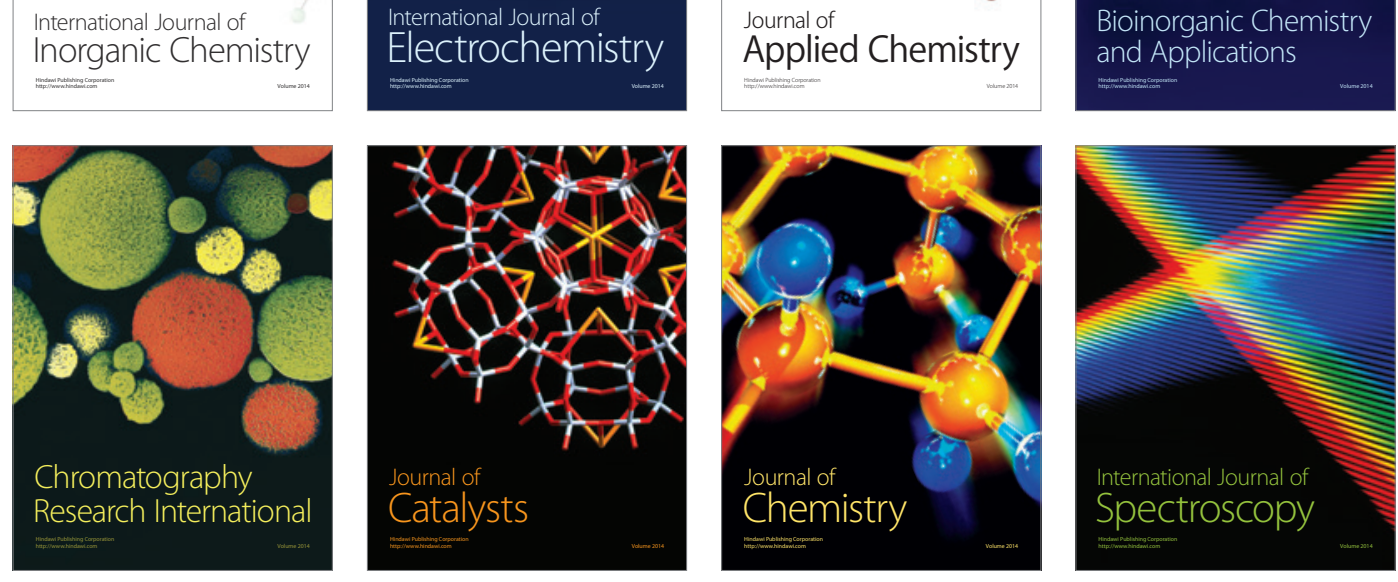